\title{
Gastric pneumatosis in a premature infant with necrotizing enterocolitis
}

Volume 6 Issue 4 - 2017

Keywords: gastric pneumatosis, preterm, infant, necrotizing enterocolitis

\section{Clinical Images}

A male infant, twin B was born at 32weeks' gestation by cesarean section due to fetal distress to a 26 year old gravida 2 and Para 1 mother. The antenatal ultrasound of our patient showed congenital heart disease. The maternal history was insignificant. He weighed 1880 grams and had Apgar scores of 9 and 9 at 1 and 5minutes respectively. He developed respiratory distress immediately after birth and required intubation and mechanical ventilation. There was no dysmorphic features. Echocardiogram and C.T cardiac with contrast showed dextrocardia, trunk's arteriosus, interrupted aortic arch, large ventricular septal defect and patent ductus arteriosus. He was clinical stable and was started on enteral feeds. He had episode of gastric aspirates but they were considered insignificant. At two weeks of age he became septic with abdominal distension. He required dobutamin to restore his low blood pressure. Abdominal radiographs showed gastric and intestinal pneumatosis without any evidence of free intraperitoneal or portal venous air (Figure 1). Leukopenia and thrombocytopenia were present. He was started on antibiotics (ampicillin, gentamicin and metronidazole). He passed bloody stools with mucous. He required several platelet transfusions to correct the severe thrombocytopenia. The septic workup showed sterile blood cultures. His abdominal radiographs improved on day five of treatment (Figure 2). His head ultrasound was normal. The clinical condition of the infant significantly improved.

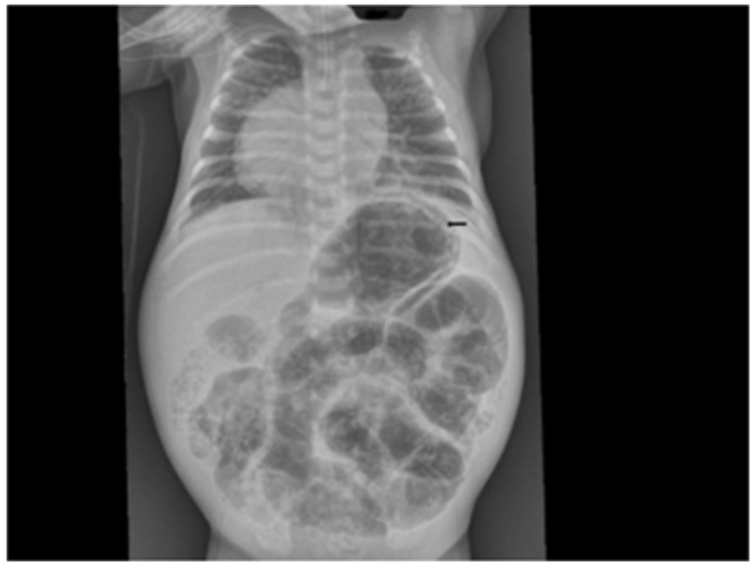

Figure I Abdominal X-ray of our patient showing gastric and intestinal pneumatosis

\section{Discussion}

Gastric pneumatosis is a rare condition and it has been found to be associated primarily with necrotizing entercolitis (NEC). It has been

\section{Saleh Al-Alaiyan, Fahad Al-Hazzani}

Department of Pediatrics, Neonatology Section, King Faisal Specialist Hospital and Research Centre, Saudi Arabia

Correspondence: Saleh Al-Alaiyan, Professor of Pediatrics, College of Medicine, AlFaisal University, Consultant Neonatologist \& King Faisal Specialist Hospital \& Research Centre, Saudi Arabia, Email alaiyan@kfshrc.edu.sa

Received: March 27, 2017| Published: March 28, 2017

considered as an indicator of intestinal perforation, which was not the case in our current patient. ${ }^{1}$ Nevertheless, gastric pneumatosis has been reported in association with pyloric or duodenal stenosis, ${ }^{2,3}$ post cardiac surgery, ${ }^{4}$ and an intramural feeding catheter. ${ }^{5}$

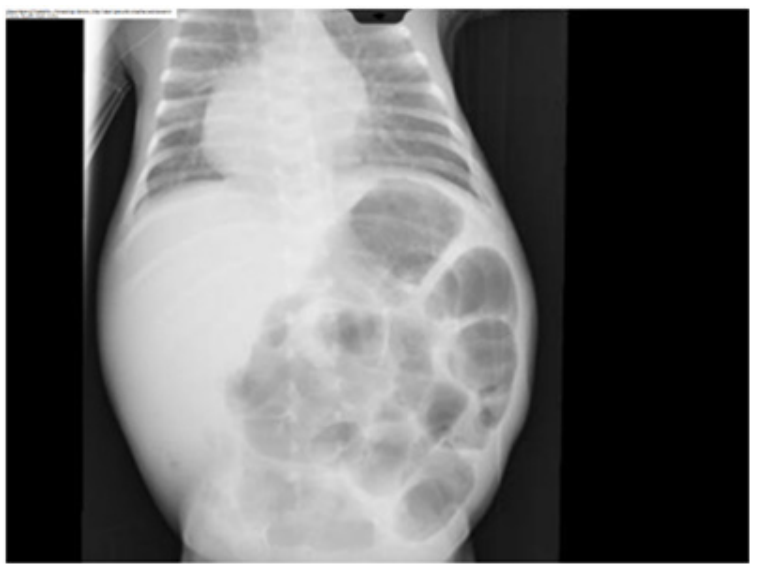

Figure 2 Abdominal X-ray of our patient after 5 days of treatment showing no gastric and intestinal pneumatosis.

The mechanism of gastric pneumatosis is not well understood. However, some reports attributed the occurrence of gastric pneumatosis to an increased gastric pressure from severe pyloric or bowel obstruction. Cohen et al has reported portal venous gas and gastric wall pneumatosis in 4 vomiting infants proven to have hypertrophic pyloric stenosis. All cases had prominent bright punctate echoes on sonography of the liver, portal vein lumen, and gastric wall. ${ }^{6}$ It has been reported in an infant following the use of noninvasive ventilation. The authors postulated that there was a mild damage in the gastric mucosa, due to the placement of the feeding tube or the use of indomethacin or both together. They believed that noninvasive 
ventilation increased the intragastric pressure that resulted in the submucosal dissection of air followed by the development of gastric pneumatosis. ${ }^{7}$ In our patient, the exact cause of gastric pneumatosis remains unclear. We postulate that cyanotic congenital heart disease caused gastrointestinal hypoperfusion and ischemia which resulted in NEC and gastric pneumatosis. A mild damage in the gastric mucosa cannot be rolled out in our case.

\section{Lesson to clinicians}

i. Gastric pneumatosis is rare but it should be suspected in infants with cyanotic congenital heart disease, feeding intolerance and abdominal distension.

ii. Prompt recognition of this condition is important for making the diagnosis and starting the appropriate management in a timely manner, to avoid more deterioration.

\section{Acknowledgments}

None.

\section{Conflicts of interest}

Author declares there are no conflicts of interest.

\section{Funding}

None.

\section{References}

1. Duran R, Vatansever U, Aksu B, et al. Gastric pneumatosis intestinalis: an indicator of intestinal perforation in preterm infants with necrotizing enterocolitis? J Pediatr Gastroenterol Nutr. 2006;43(4):539-541.

2. Holgersen LO, Borns PF, Srouji MN. Isolated gastris pneumatosis. $J$ Pediatr Surg. 1974;9:813-816.

3. Kawano S1, Tanaka H, Daimon Y, et al. Gastric pneumatosis associated with duodenal stenosis and malrotation. Pediatr Radiol. 2001;31(9):656-658.

4. Taylor D R, Tung J Y, Baffa J M, et al. Gastric pneumatosis following cardiac surgery. Eur J Pediatr . 2000;159(7):553-554.

5. Mandell G A, Finkelstein M. Gastric pneumatosis secondary to an intramural feeding catheter. Pediatr Radiol. 1988;18(5):418-420.

6. Cohen HL, Chism PB, Radtke I. Excessive Bright Echoes Sign for Hypertrophic Pyloric Stenosis Suggest the Diagnosis: Gastric Pneumatosis and Portal Venous Gas in Infants Suggest HPS. J Ultrasound Med. 2017.

7. Ting YJ, Chan K, Wong SM, et al. Gastric Pneumatosis in a Premature Neonate. AJP Reports. 2011;1(1):11-14. 